Fermilab-Pub-97/172-E

\title{
Measurement of the Top Quark Mass Using Dilepton Events
}

\author{
The D $\varnothing$ Collaboration* \\ Fermi National Accelerator Laboratory, Batavia, Illinois 60510
}

(June 9, 1997)

\begin{abstract}
The $\mathrm{D} \varnothing$ collaboration has performed a measurement of the top quark mass $m_{t}$ based on six candidate events for the process $t \bar{t} \rightarrow b W^{+} \bar{b} W^{-}$, where the $W$ bosons decay to $e \nu$ or $\mu \nu$. This sample was collected during an exposure of the $\mathrm{D} \varnothing$ detector to an integrated luminosity of $125 \mathrm{pb}^{-1}$ of $\sqrt{s}=1.8 \mathrm{TeV} p \bar{p}$ collisions. We obtain $m_{t}=168.4 \pm 12.3$ (stat) \pm 3.7 (sys) $\mathrm{GeV} / c^{2}$, consistent with the measurement obtained using single-lepton events. Combination of the single-lepton and dilepton results yields $m_{t}=172.0 \pm 7.5 \mathrm{GeV} / c^{2}$.
\end{abstract}

*Authors listed on the following page. Submitted to Physical Review Letters. 
B. Abbott,${ }^{28}$ M. Abolins, ${ }^{25}$ B.S. Acharya,${ }^{43}$ I. Adam, ${ }^{12}$ D.L. Adams, ${ }^{37}$ M. Adams, ${ }^{17}$ S. Ahn,${ }^{14}$ H. Aihara,${ }^{22}$ G.A. Alves,${ }^{10}$ E. Amidi, ${ }^{29}$ N. Amos, ${ }^{24}$ E.W. Anderson,,${ }^{19}$ R. Astur,${ }^{42}$ M.M. Baarmand, ${ }^{42}$ A. Baden, ${ }^{23}$ V. Balamurali, ${ }^{32}$ J. Balderston, ${ }^{16}$ B. Baldin,${ }^{14}$ S. Banerjee, ${ }^{43}$ J. Bantly, ${ }^{5}$ J.F. Bartlett, ${ }^{14}$ K. Bazizi ${ }^{39}$ A. Belyaev, ${ }^{26}$ S.B. Beri,${ }^{34}$ I. Bertram ${ }^{31}$ V.A. Bezzubov, ${ }^{35}$ P.C. Bhat,${ }^{14}$ V. Bhatnagar, ${ }^{34}$ M. Bhattacharjee, ${ }^{13}$ N. Biswas, ${ }^{32}$ G. Blazey,${ }^{30}$ S. Blessing, ${ }^{15}$ P. Bloom,${ }^{7}$ A. Boehnlein,${ }^{14}$ N.I. Bojko,${ }^{35}$ F. Borcherding, ${ }^{14}$ J. Borders ${ }^{39}$ C. Boswell,${ }^{9}$ A. Brandt, ${ }^{14}$ R. Brock, ${ }^{25}$ A. Bross,${ }^{14}$ D. Buchholz ${ }^{31}$ V.S. Burtovoi,${ }^{35}$ J.M. Butler ${ }^{3}$ W. Carvalho, ${ }^{10}$ D. Casey ${ }^{39}$ Z. Casilum, ${ }^{42}$ H. Castilla-Valdez ${ }^{11}$ D. Chakraborty ${ }^{42}$ S.-M. Chang, ${ }^{29}$ S.V. Chekulaev, ${ }^{35}$ L.-P. Chen,${ }^{22}$ W. Chen,${ }^{42}$ S. Choi, ${ }^{41}$ S. Chopra, ${ }^{24}$ B.C. Choudhary, ${ }^{9}$ J.H. Christenson, ${ }^{14}$ M. Chung, ${ }^{17}$ D. Claes ${ }^{27}$ A.R. Clark, ${ }^{22}$ W.G. Cobau,${ }^{23}$ J. Cochran, ${ }^{9}$ W.E. Cooper, ${ }^{14}$ C. Cretsinger, ${ }^{39}$ D. Cullen-Vidal ${ }^{5}$ M.A.C. Cummings ${ }^{16}$ D. Cutts,${ }^{5}$ O.I. Dahl,${ }^{22}$ K. Davis,${ }^{2}$ K. De ${ }^{44}$ K. Del Signore,${ }^{24}$ M. Demarteau, ${ }^{14}$ D. Denisov,${ }^{14}$ S.P. Denisov,${ }^{35}$ H.T. Diehl,${ }^{14}$ M. Diesburg, ${ }^{14}$ G. Di Loreto, ${ }^{25}$ P. Draper, ${ }^{44}$ Y. Ducros,${ }^{40}$ L.V. Dudko, ${ }^{26}$ S.R. Dugad, ${ }^{43}$ D. Edmunds,${ }^{25}$ J. Ellison, ${ }^{9}$ V.D. Elvira,${ }^{42}$ R. Engelmann, ${ }^{42}$ S. Eno, ${ }^{23}$ G. Eppley,${ }^{37}$ P. Ermolov ${ }^{26}$ O.V. Eroshin ${ }^{35}$ V.N. Evdokimov, ${ }^{35}$ T. Fahland,${ }^{8}$ M. Fatyga,${ }^{4}$ M.K. Fatyga,${ }^{39}$ J. Featherly, ${ }^{4}$ S. Feher, ${ }^{14}$ D. Fein, ${ }^{2}$ T. Ferbel,${ }^{39}$ G. Finocchiaro, ${ }^{42}$ H.E. Fisk, ${ }^{14}$ Y. Fisyak, ${ }^{7}$ E. Flattum, ${ }^{14}$ G.E. Forden,${ }^{2}$ M. Fortner,${ }^{30}$ K.C. Frame,${ }^{25}$ S. Fuess,${ }^{14}$ E. Gallas ${ }^{44}$ A.N. Galyaev ${ }^{35}$ P. Gartung, ${ }^{9}$ T.L. Geld,${ }^{25}$ R.J. Genik II,${ }^{25}$ K. Genser,${ }^{14}$ C.E. Gerber,${ }^{14}$ B. Gibbard, ${ }^{4}$ S. Glenn, ${ }^{7}$ B. Gobbi,${ }^{31}$ M. Goforth,${ }^{15}$ A. Goldschmidt,${ }^{22}$ B. Gómez ${ }^{1}$ G. Gómez, ${ }^{23}$ P.I. Goncharov, ${ }^{35}$ J.L. González Solís, ${ }^{11}$ H. Gordon, ${ }^{4}$ L.T. Goss, ${ }^{45}$ K. Gounder, ${ }^{9}$ A. Goussiou ${ }^{42}$ N. Graf, ${ }^{4}$ P.D. Grannis,${ }^{42}$ D.R. Green, ${ }^{14}$ J. Green,${ }^{30}$ H. Greenlee, ${ }^{14}$ G. Grim,${ }^{7}$ S. Grinstein,${ }^{6}$ N. Grossman, ${ }^{14}$ P. Grudberg, ${ }^{22}$ S. Grünendahl,${ }^{39}$ G. Guglielmo, ${ }^{33}$ J.A. Guida, ${ }^{2}$ J.M. Guida,${ }^{5}$ A. Gupta,${ }^{43}$ S.N. Gurzhiev, ${ }^{35}$ P. Gutierrez ${ }^{33}$ Y.E. Gutnikov, ${ }^{35}$ N.J. Hadley, ${ }^{23}$ H. Haggerty, ${ }^{14}$ S. Hagopian, ${ }^{15}$ V. Hagopian, ${ }^{15}$ K.S. Hahn,${ }^{39}$ R.E. Hall, ${ }^{8}$ S. Hansen, ${ }^{14}$ J.M. Hauptman, ${ }^{19}$ D. Hedin,${ }^{30}$ A.P. Heinson, ${ }^{9}$ U. Heintz,${ }^{14}$ R. Hernández-Montoya, ${ }^{11}$ T. Heuring, ${ }^{15}$ R. Hirosky,${ }^{15}$ J.D. Hobbs,${ }^{14}$ B. Hoeneisen, ${ }^{1, \dagger}$ J.S. Hoftun,${ }^{5}$ F. Hsieh, ${ }^{24}$ Ting Hu,${ }^{42}$ Tong Hu, ${ }^{18}$ T. Huehn,${ }^{9}$ A.S. Ito, ${ }^{14}$ E. James, ${ }^{2}$ J. Jaques, ${ }^{32}$ S.A. Jerger, ${ }^{25}$ R. Jesik, ${ }^{18}$ J.Z.-Y. Jiang, ${ }^{42}$ T. Joffe-Minor, ${ }^{31}$ K. Johns, ${ }^{2}$ M. Johnson, ${ }^{14}$ A. Jonckheere, ${ }^{14}$ M. Jones,${ }^{16}$ H. Jöstlein, ${ }^{14}$ S.Y. Jun,${ }^{31}$ C.K. Jung, ${ }^{42}$ S. Kahn, ${ }^{4}$ G. Kalbfleisch, ${ }^{33}$ J.S. Kang, ${ }^{20}$ R. Kehoe, ${ }^{32}$ M.L. Kelly, ${ }^{32}$ C.L. Kim, ${ }^{20}$ S.K. Kim, ${ }^{41}$ A. Klatchko, ${ }^{15}$ B. Klima, ${ }^{14}$ C. Klopfenstein, ${ }^{7}$ V.I. Klyukhin, ${ }^{35}$ V.I. Kochetkov, ${ }^{35}$ J.M. Kohli, ${ }^{34}$ D. Koltick, ${ }^{36}$ A.V. Kostritskiy, ${ }^{35}$ J. Kotcher, ${ }^{4}$ A.V. Kotwal, ${ }^{12}$ J. Kourlas, ${ }^{28}$ A.V. Kozelov, ${ }^{35}$ E.A. Kozlovski ${ }^{35}$ J. Krane,${ }^{27}$ M.R. Krishnaswamy, ${ }^{43}$ S. Krzywdzinski,${ }^{14}$ S. Kunori,${ }^{23}$ S. Lami, ${ }^{42}$ H. Lan,${ }^{14, *}$ R. Lander, ${ }^{7}$ F. Landry, ${ }^{25}$ G. Landsberg, ${ }^{14}$ B. Lauer, ${ }^{19}$ A. Leflat,${ }^{26} \mathrm{H}$. Li,${ }^{42} \mathrm{~J}$. Li, ${ }^{44}$ Q.Z. Li-Demarteau, ${ }^{14}$ J.G.R. Lima,${ }^{38}$ D. Lincoln,${ }^{24}$ S.L. Linn,${ }^{15}$ J. Linnemann,${ }^{25}$ R. Lipton, ${ }^{14}$ Q. Liu,,${ }^{14, *}$ Y.C. Liu ${ }^{31}$ F. Lobkowicz, ${ }^{39}$ S.C. Loken ${ }^{22}$ S. Lökös, ${ }^{42}$ L. Lueking, ${ }^{14}$ A.L. Lyon, ${ }^{23}$ A.K.A. Maciel,${ }^{10}$ R.J. Madaras,${ }^{22}$ R. Madden, ${ }^{15}$ L. Magaña-Mendoza, ${ }^{11}$ S. Mani, ${ }^{7}$ H.S. Mao,${ }^{14, *}$ R. Markeloff,${ }^{30}$ L. Markosky, ${ }^{2}$ T. Marshall, ${ }^{18}$ M.I. Martin, ${ }^{14}$ K.M. Mauritz, ${ }^{19}$ B. May, ${ }^{31}$ A.A. Mayorov, ${ }^{35}$ R. McCarthy, ${ }^{42}$ J. McDonald, ${ }^{15}$ T. McKibben, ${ }^{17}$ J. McKinley, ${ }^{25}$ T. McMahon, ${ }^{33}$ H.L. Melanson, ${ }^{14}$ M. Merkin,${ }^{26}$ K.W. Merritt, ${ }^{14}$ H. Miettinen,${ }^{37}$ A. Mincer, ${ }^{28}$ J.M. de Miranda, ${ }^{10}$ C.S. Mishra, ${ }^{14}$ N. Mokhov,${ }^{14}$ N.K. Mondal,${ }^{43}$ H.E. Montgomery ${ }^{14}$ P. Mooney, ${ }^{1}$ H. da Motta,${ }^{10}$ C. Murphy, ${ }^{17}$ F. Nang, ${ }^{2}$ M. Narain,,${ }^{14}$ V.S. Narasimham ${ }^{43}$ A. Narayanan, ${ }^{2}$ H.A. Neal, ${ }^{24}$ J.P. Negret,,${ }^{1}$ P. Nemethy, ${ }^{28}$ M. Nicola,,${ }^{10}$ 
D. Norman ${ }^{45}$ L. Oesch,${ }^{24}$ V. Oguri,${ }^{38}$ E. Oltman,${ }^{22}$ N. Oshima, ${ }^{14}$ D. Owen, ${ }^{25}$ P. Padley,${ }^{37}$ M. Pang, ${ }^{19}$ A. Para,${ }^{14}$ Y.M. Park,${ }^{21}$ R. Partridge,${ }^{5}$ N. Parua,${ }^{43}$ M. Paterno ${ }^{39}$ J. Perkins, ${ }^{44}$ M. Peters, ${ }^{16}$ R. Piegaia,${ }^{6}$ H. Piekarz ${ }^{15}$ Y. Pischalnikov, ${ }^{36}$ V.M. Podstavkov ${ }^{35}$ B.G. Pope,${ }^{25}$ H.B. Prosper, ${ }^{15}$ S. Protopopescu, ${ }^{4}$ J. Qian,${ }^{24}$ P.Z. Quintas,${ }^{14}$ R. Raja,${ }^{14}$ S. Rajagopalan,${ }^{4}$ O. Ramirez, ${ }^{17}$ L. Rasmussen, ${ }^{42}$ S. Reucroft, ${ }^{29}$ M. Rijssenbeek, ${ }^{42}$ T. Rockwell,${ }^{25}$ N.A. Roe ${ }^{22}$ P. Rubinov, ${ }^{31}$ R. Ruchti, ${ }^{32}$ J. Rutherfoord,${ }^{2}$ A. Sánchez-Hernández, ${ }^{11}$ A. Santoro, ${ }^{10}$

L. Sawyer, ${ }^{44}$ R.D. Schamberger, ${ }^{42}$ H. Schellman, ${ }^{31}$ J. Sculli, ${ }^{28}$ E. Shabalina,,${ }^{26}$ C. Shaffer,${ }^{15}$ H.C. Shankar, ${ }^{43}$ R.K. Shivpuri, ${ }^{13}$ M. Shupe,${ }^{2}$ H. Singh,${ }^{9}$ J.B. Singh,${ }^{34}$ V. Sirotenko, ${ }^{30}$ W. Smart,${ }^{14}$ A. Smith, ${ }^{2}$ R.P. Smith,${ }^{14}$ R. Snihur, ${ }^{31}$ G.R. Snow, ${ }^{27}$ J. Snow ${ }^{33}$ S. Snyder, ${ }^{4}$ J. Solomon, ${ }^{17}$ P.M. Sood ${ }^{34}$ M. Sosebee,${ }^{44}$ N. Sotnikova, ${ }^{26}$ M. Souza, ${ }^{10}$ A.L. Spadafora,${ }^{22}$ R.W. Stephens, ${ }^{44}$ M.L. Stevenson, ${ }^{22}$ D. Stewart,${ }^{24}$ D.A. Stoianova, ${ }^{35}$ D. Stoker, ${ }^{8}$ M. Strauss, ${ }^{33}$ K. Streets, ${ }^{28}$ M. Strovink ${ }^{22}$ A. Sznajder, ${ }^{10}$ P. Tamburello, ${ }^{23}$ J. Tarazi, ${ }^{8}$ M. Tartaglia, ${ }^{14}$ T.L.T. Thomas, ${ }^{31}$ J. Thompson,${ }^{23}$ T.G. Trippe, ${ }^{22}$ P.M. Tuts,${ }^{12}$ N. Varelas ${ }^{25}$ E.W. Varnes, ${ }^{22}$ D. Vititoe,${ }^{2}$ A.A. Volkov, ${ }^{35}$ A.P. Vorobiev,${ }^{35}$ H.D. Wahl,${ }^{15}$ G. Wang ${ }^{15}{ }^{15}$ J. Warchol, ${ }^{32}$ G. Watts, ${ }^{5}$ M. Wayne,${ }^{32}$ H. Weerts ${ }^{25}$ A. White, ${ }^{44}$ J.T. White ${ }^{45}$ J.A. Wightman ${ }^{19}{ }^{\text {S. Willis }},{ }^{30}$ S.J. Wimpenny, ${ }^{9}$ J.V.D. Wirjawan, ${ }^{45}$ J. Womersley ${ }^{14}{ }^{14}$ E. Won,${ }^{39}$ D.R. Wood,${ }^{29}$ H. Xu,${ }^{5}$ R. Yamada,${ }^{14}$ P. Yamin, ${ }^{4}$ C. Yanagisawa, ${ }^{42}$ J. Yang,${ }^{28}$ T. Yasuda, ${ }^{29}$ P. Yepes ${ }^{37}$ C. Yoshikawa, ${ }^{16}$ S. Youssef,${ }^{15}$ J. Yu ${ }^{14}$ Y. Yu,${ }^{41}$ Z.H. Zhu,${ }^{39}$ D. Zieminska, ${ }^{18}$ A. Zieminski, ${ }^{18}$ E.G. Zverev, ${ }^{26}$ and A. Zylberstejn ${ }^{40}$

(DØ Collaboration) 


\author{
${ }^{1}$ Universidad de los Andes, Bogotá, Colombia \\ ${ }^{2}$ University of Arizona, Tucson, Arizona 85721 \\ ${ }^{3}$ Boston University, Boston, Massachusetts 02215 \\ ${ }^{4}$ Brookhaven National Laboratory, Upton, New York 11973 \\ ${ }^{5}$ Brown University, Providence, Rhode Island 02912 \\ ${ }^{6}$ Universidad de Buenos Aires, Buenos Aires, Argentina \\ ${ }^{7}$ University of California, Davis, California 95616 \\ ${ }^{8}$ University of California, Irvine, California 92697 \\ ${ }^{9}$ University of California, Riverside, California 92521 \\ ${ }^{10}$ LAFEX, Centro Brasileiro de Pesquisas Físicas, Rio de Janeiro, Brazil \\ ${ }^{11}$ CINVESTAV, Mexico City, Mexico \\ ${ }^{12}$ Columbia University, New York, New York 10027 \\ ${ }^{13}$ Delhi University, Delhi, India 110007 \\ ${ }^{14}$ Fermi National Accelerator Laboratory, Batavia, Illinois 60510 \\ ${ }^{15}$ Florida State University, Tallahassee, Florida 32306 \\ ${ }^{16}$ University of Hawaii, Honolulu, Hawaii 96822 \\ ${ }^{17}$ University of Illinois at Chicago, Chicago, Illinois 60607 \\ ${ }^{18}$ Indiana University, Bloomington, Indiana 47405 \\ ${ }^{19}$ Iowa State University, Ames, Iowa 50011 \\ ${ }^{20}$ Korea University, Seoul, Korea \\ ${ }^{21}$ Kyungsung University, Pusan, Korea \\ ${ }^{22}$ Lawrence Berkeley National Laboratory and University of California, Berkeley, California 94720 \\ ${ }^{23}$ University of Maryland, College Park, Maryland 20742 \\ ${ }^{24}$ University of Michigan, Ann Arbor, Michigan 48109 \\ ${ }^{25}$ Michigan State University, East Lansing, Michigan 48824 \\ ${ }^{26}$ Moscow State University, Moscow, Russia \\ ${ }^{27}$ University of Nebraska, Lincoln, Nebraska 68588 \\ ${ }^{28}$ New York University, New York, New York 10003 \\ ${ }^{29}$ Northeastern University, Boston, Massachusetts 02115 \\ ${ }^{30}$ Northern Illinois University, DeKalb, Illinois 60115 \\ ${ }^{31}$ Northwestern University, Evanston, Illinois 60208 \\ ${ }^{32}$ University of Notre Dame, Notre Dame, Indiana 46556 \\ ${ }^{33}$ University of Oklahoma, Norman, Oklahoma 73019 \\ ${ }^{34}$ University of Panjab, Chandigarh 16-00-14, India \\ ${ }^{35}$ Institute for High Energy Physics, 142-284 Protvino, Russia \\ ${ }^{36}$ Purdue University, West Lafayette, Indiana 47907 \\ ${ }^{37}$ Rice University, Houston, Texas 77005 \\ ${ }^{38}$ Universidade Estadual do Rio de Janeiro, Brazil \\ ${ }^{39}$ University of Rochester, Rochester, New York 14627 \\ ${ }^{40}$ CEA, DAPNIA/Service de Physique des Particules, CE-SACLAY, Gif-sur-Yvette, France \\ ${ }^{41}$ Seoul National University, Seoul, Korea \\ ${ }^{42}$ State University of New York, Stony Brook, New York 11794 \\ ${ }^{43}$ Tata Institute of Fundamental Research, Colaba, Mumbai 400005, India \\ ${ }^{44}$ University of Texas, Arlington, Texas 76019 \\ ${ }^{45}$ Texas A $6 M$ University, College Station, Texas 77843
}


The pair production of top quarks has been observed in $p \bar{p}$ collisions at $\sqrt{\mathrm{s}}=1.8 \mathrm{TeV}$ by the CDF and DØ collaborations [1]. Since the time of observation, the integrated luminosity has more than doubled (to $125 \mathrm{pb}^{-1}$ ) and the $\mathrm{D} \varnothing$ experiment has substantially improved its techniques for measurement of the top quark mass $m_{t}$. We previously reported a measurement of $m_{t}$ using events in which one top quark decayed semileptonically and the other decayed hadronically (the " $\ell+$ jets" mode, where $\ell=e$ or $\mu$ ), giving $m_{t}=173.3 \pm 5.6$ (stat) \pm 6.2 (sys) GeV/c [2]. This letter reports a first measurement of $m_{t}$ using events consistent with the $t \bar{t} \rightarrow b W^{+} \bar{b} W^{-} \rightarrow b \ell^{+} \nu \bar{b} \ell^{-} \bar{\nu}$ ("dilepton") hypothesis. This independent measurement is important as a direct test of the hypothesis that the excess of events over background in both the $\ell+$ jets and dilepton channels is due to $t \bar{t}$ production.

The events used in this analysis were recorded by the D $\varnothing$ detector [3], which consists of a nonmagnetic tracking system, including a transition radiation detector (TRD), surrounded by a hermetic liquid argon/uranium calorimeter, segmented in depth into several electromagnetic (EM) and hadronic layers, and an outer toroidal muon spectrometer. Electrons are identified using a likelihood method based on the EM shower shape, track ionization, spatial match of the track with the EM shower, and TRD response. Muons are required to have reconstructed tracks in the central tracking chamber and in at least one of the spectrometer layers outside of the toroid, and to have energy deposition in the calorimeter consistent with the passage of a minimum ionizing particle. Jets are reconstructed from calorimeter energy clustered within a cone of radius $\sqrt{(\Delta \eta)^{2}+(\Delta \phi)^{2}}=0.5$ 《4].

We select dilepton top candidate events according to criteria similar to those used in our cross section measurement [5]. This selection requires two leptons, with the transverse energy $E_{T}$ of each lepton $>15(20) \mathrm{GeV}$ for the $e \mu$ and $\mu \mu(e e)$ channels, with $\left|\eta_{e}\right|<2.5$ and $\left|\eta_{\mu}\right|<1.7$, and two or more jets with $E_{T}>20 \mathrm{GeV}$ and $|\eta|<2.5$. In addition, for the $e e$ and $e \mu$ channels we require significant missing transverse energy $\mathbb{E}_{T}$ to discriminate against background sources that have no final-state neutrinos, while for the $\mu \mu$ analysis we reduce the $Z$ boson background by rejecting events for which the $\chi^{2}$ probability of a fit to the $Z \rightarrow \mu \mu$ hypothesis is $>1 \%$. We reject much of the remaining background using the quantities $H_{T} \equiv \sum_{\text {jets }} E_{T}$ and $H_{T}^{e} \equiv H_{T}+E_{T}\left(e_{1}\right)$, where all jets with $E_{T}>15 \mathrm{GeV}$ and $|\eta|<2.5$ enter the sum, and $e_{1}$ is the leading $E_{T}$ electron. The selection requires $H_{T}^{e}\left(H_{T}\right)>120(100) \mathrm{GeV}$ for the ee and $e \mu(\mu \mu)$ channels. The ee selection reported in Ref. [5] is extended to include an event that contains, in addition to an electron, one EM energy cluster without an associated reconstructed track, but with hits in the layers of the central tracking system between the interaction vertex and the cluster. This event also has a muon near one of its jets, which is evidence of $b$ quark decay, and further enhances the probability that the event is an example of $t \bar{t}$ production. The signal to background ratio for such $b$-tagged events in which only one of the EM clusters has an associated track is $\approx$ 15/1. The final sample therefore consists of three $e \mu$ events, two ee events, and one $\mu \mu$ event, with expected backgrounds of $0.21 \pm 0.16,0.47 \pm 0.09$, and $0.73 \pm 0.25$ events, respectively (background sources are detailed in Ref [5]). Kinematic details for the observed events can be found in Ref. [6].

We reconstruct the events according to the $t \bar{t}$ dilepton decay hypothesis. After applying the invariant mass constraints $m\left(\ell_{1} \nu_{1}\right)=m\left(\ell_{2} \nu_{2}\right)=m_{W}$ and $m\left(\ell_{1} \nu_{1} b_{1}\right)=m\left(\ell_{2} \nu_{2} b_{2}\right)$, the system remains underconstrained due to the two undetected neutrinos. We supply the 
needed additional constraints by assuming values for $m_{t}$ and for two quantities associated with the neutrinos (as discussed below). We then solve for the neutrino momenta up to a fourfold ambiguity and assign a weight to each solution to characterize how likely it is to occur in $t \bar{t}$ production for the assumed $m_{t}$ [0]. We compute the relative weight as a function of assumed $m_{t}$ for $80<m_{t}<280 \mathrm{GeV} / c^{2}$, employing two weighting schemes with differing sensitivities to top production kinematics, decay distributions, and phase space volume consistent with the event topology.

The first weighting scheme is called matrix element weighting $(\mathcal{M W T})$, and is an extension of the procedure given in Ref. [8]. Here, we require the sum of the neutrino $\vec{p}_{T}$ 's to equal the measured $\vec{H}_{T}$. We assign a weight

$$
W_{o}\left(m_{t}\right)=A\left(m_{t}\right) f(x) f(\bar{x}) P\left(E_{\ell 1}^{C M} \mid m_{t}\right) P\left(E_{\ell 2}^{C M} \mid m_{t}\right)
$$

where $f(x)$ is the CTEQ3M [9] parton distribution function evaluated at the proton (antiproton) momentum fraction $x(\bar{x})$ required by the solution, and $P\left(E_{\ell}^{C M} \mid m_{t}\right)$ is the probability density for the lepton energy in the top quark rest frame. The factor $A\left(m_{t}\right)$ normalizes the average weight of accepted events to unity, independent of the top quark mass. For each mass, we add the weights of all solutions.

The other weighting scheme, called neutrino weighting $(\nu \mathrm{WT})$ [6], steps the assumed $\eta$ for each neutrino through a range of values at each $m_{t}$. Each step spans an equal fraction of the neutrino $\eta$ distribution expected in $t \bar{t}$ production. At each step a weight is assigned based on the extent to which the $\vec{E}_{T}$ measured in the event agrees with the sum of the neutrino $\vec{p}_{T}$ 's in the solution. The Gaussian resolution of each component of the $\vec{H}_{T}$ is 4 $\mathrm{GeV}$. The weights at all $\eta$ values are summed to give $W_{o}\left(m_{t}\right)$ in this method.

In calculating the consistency of the event kinematics with any given $m_{t}$, we also account for detector resolution for jets, leptons, and $\mathbb{E}_{T}$. This is done by fluctuating all the measured energies by their resolutions 100 (5000) times for MC (collider data) events and summing the weights obtained for each fluctuation. The Gaussian resolutions for electrons and muons are $\sigma_{E} / E=15 \% / \sqrt{E} \oplus 3 \%$ and $\sigma(1 / p)=0.18(p-2) / p^{2} \oplus 0.003$, respectively, with $E(p)$ in $\mathrm{GeV}(\mathrm{GeV} / c)$. Jets are smeared by double Gaussians designed to model both the inherent energy resolution of the hadronic calorimeter (narrow Gaussian) and the contribution of large angle gluon radiation to the resolution (wide Gaussian). The $\overrightarrow{\mathscr{H}}_{T}$ is then recomputed to reflect the changes in jet and lepton energies, and each component is fluctuated with a 4 $\mathrm{GeV}$ Gaussian.

For both the $\mathcal{M W T}$ and $\nu \mathrm{WT}$ methods, the up to fourfold solution ambiguity is handled by summing the weights for all solutions. There remains a twofold ambiguity in the pairing of jets to leptons in reconstructing the event. Both analyses sum over both pairings. In addition, initial- and final-state gluon radiation (ISR and FSR) can create extra jets that further complicate the final state. For events with more than two jets, a weighted sum is taken over all possible combinations of the three leading $E_{T}$ jets. If jet $i$ is assumed to arise from ISR, then the weight is given by $\exp \left[-E_{T} \sin \theta_{i} /(25 \mathrm{GeV})\right]$ [ [4]. If jets $i$ and $j$ are assumed to arise from the same $b$ quark by FSR and have invariant mass $m_{i j}$, the weight assigned is $\exp \left[-m_{i j} /\left(20 \mathrm{GeV} / c^{2}\right)\right]$. In each case, the form of the weight is based on the characteristics of gluon radiation and the coefficient is determined empirically to maximize the sensitivity. The distributions of $W\left(m_{t}\right)$ (which corresponds to $W_{o}\left(m_{t}\right)$ after accounting 


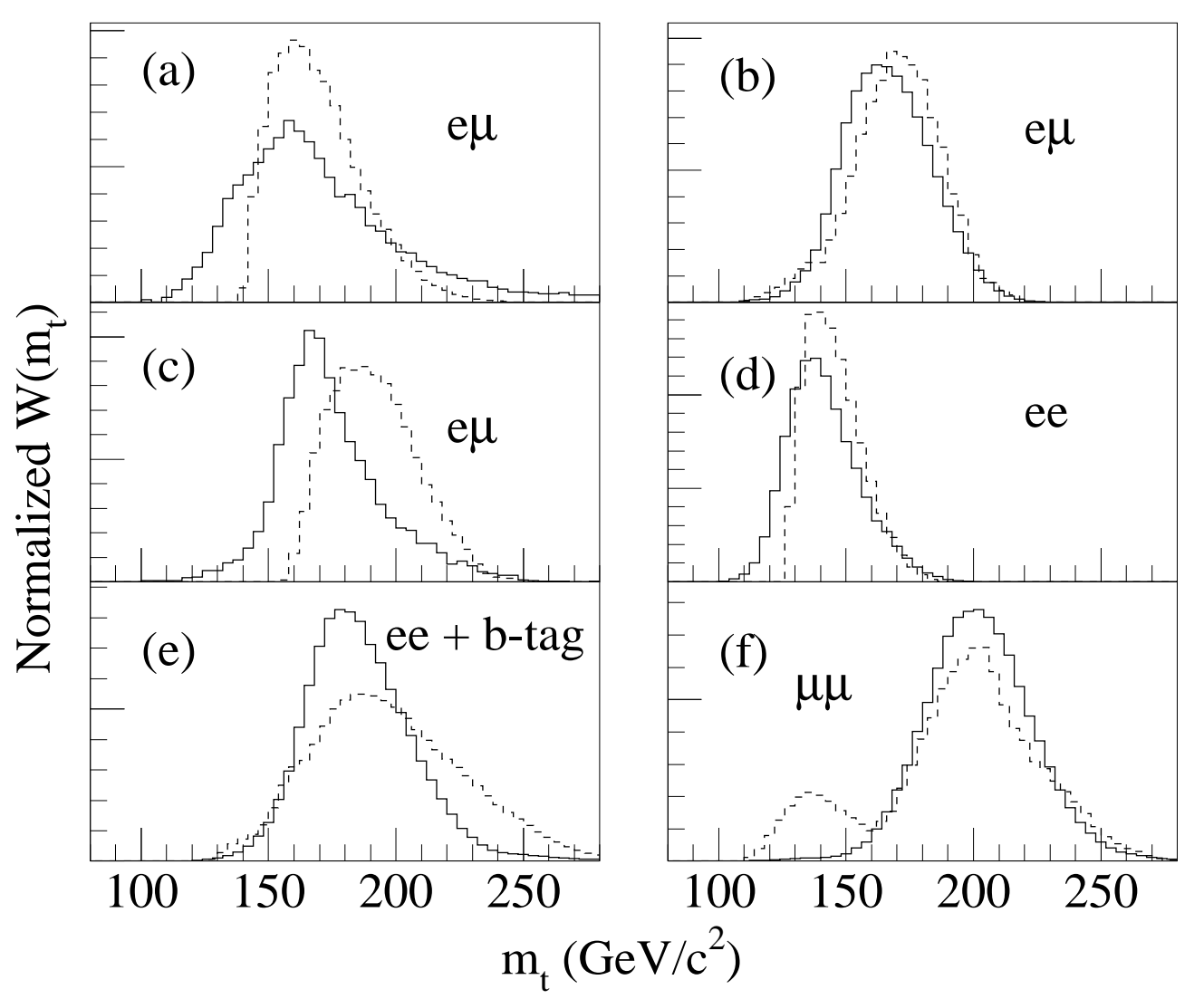

FIG. 1. $W\left(m_{t}\right)$ distributions for the six dilepton candidates, using the $\mathcal{M W T}$ (dashed) and $\nu \mathrm{WT}$ (solid) methods.

for resolution and jet combinations) for the six candidate events, using the two weighting methods, are displayed in Fig. 11.

Due to combinatorics, and the fact that no account has been taken of background, the $W\left(m_{t}\right)$ distribution derived from either the $\mathcal{M W T}$ or $\nu \mathrm{WT}$ method cannot be considered to be a probability density. Consequently, a maximum likelihood fit is performed in which the $W\left(m_{t}\right)$ distributions for data are compared to the expectations from signal and background. The signal is modeled using HERWIG [10] while the various background sources are modeled by ISAJET [11], PYTHIA [12], and D $\varnothing$ data (for the instrumental backgrounds) [5].

For both analyses, the maximum likelihood fit proceeds by normalizing the $W\left(m_{t}\right)$ distribution for each event to unity, and integrating the fractional weights in five $40 \mathrm{GeV} / c^{2}$ bins in $m_{t}$. The first four bins form the components of a four-dimensional vector $\vec{w}_{i}$ for each event $i$. Using the shape of the $W\left(m_{t}\right)$ distributions increases the statistical sensitivity of the measurement by $\approx 25 \%$ over a fit to a single-valued mass estimator for each event. The likelihood $L\left(m_{t}, n_{s}, n_{b}\right)$ to be maximized is

$$
L=g\left(n_{b}\right) p\left(n_{s}+n_{b}\right) \times \prod_{i}^{N} \frac{n_{s} f_{s}\left(\vec{w}_{i} \mid m_{t}\right)+n_{b} f_{b}\left(\vec{w}_{i}\right)}{n_{s}+n_{b}},
$$

where $n_{s}$ and $n_{b}$ are the fitted signal and background levels, $g\left(n_{b}\right)$ is a Gaussian constraint that $n_{b}$ be consistent with expectations, $p\left(n_{s}+n_{b}\right)$ is a Poisson constraint that $n_{s}+n_{b}$ be 
TABLE I. Summary of $m_{t}$ measurements for full and partial data sets. Uncertainties are statistical only.

\begin{tabular}{lcc}
\hline \hline Channels Fit & $\mathcal{M W T}$ & $\nu \mathrm{WT}$ \\
\hline$e \mu+e e+\mu \mu$ & $168.1 \pm 12.4 \mathrm{GeV} / c^{2}$ & $169.9 \pm 14.8 \mathrm{GeV} / c^{2}$ \\
$e \mu+e e$ & $167.9 \pm 12.6 \mathrm{GeV} / c^{2}$ & $173.2 \pm 14.0 \mathrm{GeV} / c^{2}$ \\
$e \mu$ & $173.1 \pm 13.3 \mathrm{GeV} / c^{2}$ & $170.1 \pm 14.5 \mathrm{GeV} / c^{2}$ \\
\hline \hline
\end{tabular}

consistent with the sample size $N$, and $f_{s}$ and $f_{b}$ are the four-dimensional probability densities for signal and background. The probability densities $f(\vec{w})$ are estimated by summing the contributions of four-dimensional Gaussian kernels placed at the location of $\vec{w}$ for each event in the signal MC or background samples [13]. Using the estimated $f_{s}$ and $f_{b}, L$ is maximized with respect to $n_{s}$ and $n_{b}$ for each value of $m_{t}$ at which we have generated MC events. The maximum likelihood estimate of $m_{t}\left(\widehat{m}_{t}\right)$ and its error $(\hat{\sigma})$ are determined by a quadratic fit to $-\ln L$ for the nine points about the minimum.

Applying the maximum likelihood fit to the data, we determine the top quark mass to be $m_{t}=168.1 \pm 12.4 \mathrm{GeV} / c^{2}(\mathcal{M W T})$, and $m_{t}=169.9 \pm 14.8 \mathrm{GeV} / c^{2}(\nu \mathrm{WT})$, where the uncertainties are statistical only (see Fig. 2). The results of fits to subsamples of the data are listed in Table $\llbracket$.

To study the properties of our mass estimator, $1000 \mathrm{MC}$ "experiments" are generated by randomly selecting three $e \mu$, two $e e$, and one $\mu \mu$ events from simulated top (with input mass $m_{t}^{M C}$ ) and background samples, according to the estimated background contamination. Due to the small sample size the estimator is not normally distributed. Figure 3 shows a typical distribution for $\widehat{m}_{t}$. The distribution is characterized by its median and width (half of the shortest interval that contains $68.3 \%$ of the experiments). The width of a Gaussian fit to the pull distribution $\left(\widehat{m}_{t}-m_{t}^{M C}\right) / \hat{\sigma}$ is consistent with unity. This verifies that $\hat{\sigma}$ is an unbiased estimate of the statistical uncertainty. The pulls means can differ from zero because of the non-Gaussian tails of the $\widehat{m}_{t}$ distribution. Table II lists medians, widths, and means and widths of the pulls for different $m_{t}^{M C}$. The properties of $\widehat{m}_{t}$ are very similar for both methods.

The sources of systematic uncertainty are summarized in Table [II]. A major uncertainty arises from the jet energy calibration, which proceeds in two steps. The first step uses $\gamma+$ jets events to relate the well-calibrated electromagnetic scale to the hadronic scale. Effects con-

TABLE II. Properties of the maximum likelihood estimate $\widehat{m}_{t}$.

\begin{tabular}{cclccc}
\hline \hline \multirow{2}{*}{$\begin{array}{c}m_{t}^{M C} \\
\mathrm{GeV} / c^{2}\end{array}$} & $\begin{array}{c}\text { Median } \\
\mathrm{GeV} / c^{2}\end{array}$ & $\begin{array}{c}\text { Width } \\
\mathrm{GeV} / c^{2}\end{array}$ & $\begin{array}{c}\text { Pull } \\
\text { Mean }\end{array}$ & $\begin{array}{c}\text { Pull } \\
\text { Width }\end{array}$ \\
\hline \multirow{3}{*}{$\mathcal{M W T}$} & 160 & 161.6 & 15.8 & $0.12 \pm 0.03$ & 1.03 \\
& 170 & 172.2 & 16.7 & $0.11 \pm 0.03$ & 0.99 \\
& 180 & 180.5 & 17.3 & $0.00 \pm 0.03$ & 0.98 \\
\hline \multirow{3}{*}{$\nu \mathrm{WT}$} & 160 & 161.5 & 14.4 & $0.17 \pm 0.03$ & 0.96 \\
& 170 & 172.2 & 16.2 & $0.08 \pm 0.03$ & 0.98 \\
& 180 & 180.5 & 18.1 & $0.03 \pm 0.03$ & 1.03 \\
\hline \hline
\end{tabular}




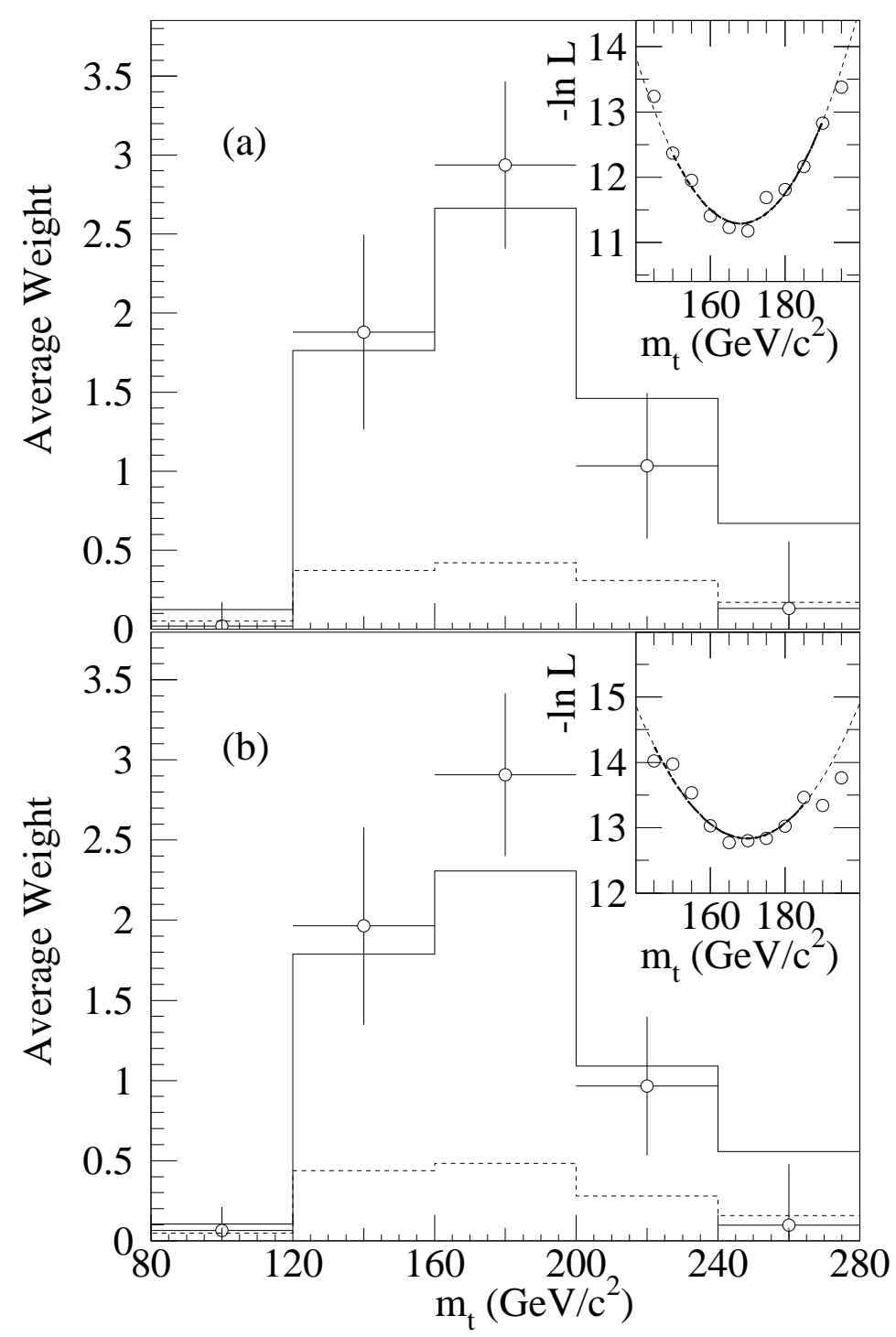

FIG. 2. Sum of the normalized candidate weights grouped into the five bins considered in the maximum likelihood fit (circles) for the (a) $\mathcal{M W T}$ and (b) $\nu \mathrm{WT}$ analyses. The uncertainty on these points is taken from the RMS spread of the weights in MC studies. Also shown are the average weights from the best-fit background (dashed) and signal plus background (solid). The $-\ln L$ distributions and quadratic fits are inset.

sidered include the overall hadronic response, energy added to jets by multiple interactions and uranium noise, and the spread of the hadronic shower outside of the jet cone [14]. The second step follows from a detailed comparison of $\gamma+$ jets events in MC and collider data, providing a correction that depends on jet $\eta$ and ensures that the energy scale in data matches that in MC. An additional correction is applied to jets having a muon within the jet cone. Under the assumption that the muon was produced in the semileptonic decay of a 


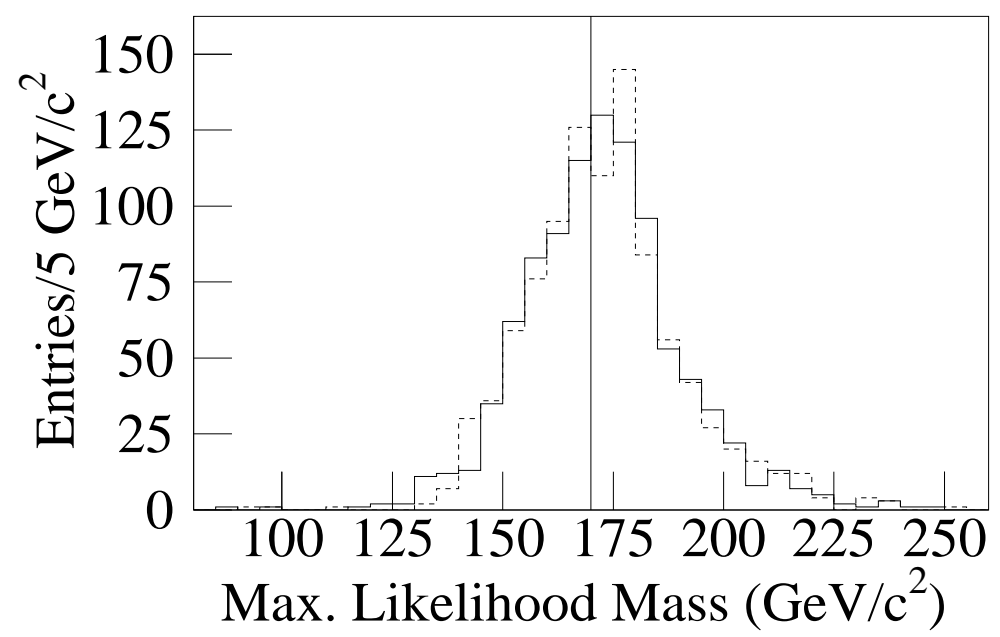

FIG. 3. Distribution of $\widehat{m}_{t}$ for "experiments" with $m_{t}^{M C}=170 \mathrm{GeV} / c^{2}$ (marked by vertical line) using the $\mathcal{M W T}$ (dashed) and $\nu \mathrm{WT}$ (solid) analysis methods.

TABLE III. Systematic errors in the measurement of $m_{t}$.

\begin{tabular}{lc}
\hline \hline Source & Error $\left(\mathrm{GeV} / c^{2}\right)$ \\
\hline Jet energy scale & 2.4 \\
Signal model & 1.8 \\
Multiple interactions & 1.3 \\
Background model & 1.2 \\
Likelihood fit & 1.3 \\
\hline Total & 3.7 \\
\hline \hline
\end{tabular}

heavy quark, the jet energy is increased take into account the muon and neutrino energies. After these corrections, we find (using the $\gamma+$ jets and smaller $Z(\rightarrow e e)+$ jets samples) that the data and MC scales agree, with an uncertainty of $\delta\left(E_{T}\right)=0.025 E_{T}+0.5 \mathrm{GeV}$. MC tests are run on samples with the jet energies rescaled by $\pm \delta$ to yield an energy scale uncertainty of $2.4 \mathrm{GeV} / c^{2}$ in $m_{t}$.

Other sources of uncertainty arise from differences among models of $t \bar{t}$ and background production, multiple interactions, and the likelihood fit procedure. ISAJET is used as a crosscheck of the HERWIG $t \bar{t}$ production model. The effect of multiple interactions is estimated using MC samples with additional random $p \bar{p}$ interactions overlaid on $t \bar{t}$ production. The contributions from all sources are summed in quadrature to give the systematic uncertainty on the measurement (see Table III).

Taking account of the $77 \%$ correlation between the $\mathcal{M W T}$ and $\nu \mathrm{WT}$ analyses, we measure the mass of the top quark in the dilepton channel by combining the two results:

$$
m_{t}=168.4 \pm 12.3 \text { (stat) } \pm 3.7 \text { (sys) } \mathrm{GeV} / c^{2} .
$$

This value is in good agreement with the measurement from the $\ell+$ jets channel [2], consistent with the $t \bar{t}$ hypothesis for both channels. We combine the results of the single- and 
dilepton analyses by propagating the systematic uncertainties in each channel with correlation coefficients of either zero (for MC statistics and background model) or unity (for jet energy scale, multiple interactions, and $t \bar{t}$ production models). Using all dilepton and $\ell+$ jets $t \bar{t}$ candidates, we measure:

$$
m_{t}=172.0 \pm 5.1 \text { (stat) } \pm 5.5 \text { (sys) } \mathrm{GeV} / c^{2}
$$

or, combining statistical and systematic errors in quadrature, $m_{t}=172.0 \pm 7.5 \mathrm{GeV} / \mathrm{c}^{2}$.

We thank the staffs at Fermilab and collaborating institutions for their contributions to this work, and acknowledge support from the Department of Energy and National Science Foundation (U.S.), Commissariat à L'Energie Atomique (France), State Committee for Science and Technology and Ministry for Atomic Energy (Russia), CNPq (Brazil), Departments of Atomic Energy and Science and Education (India), Colciencias (Colombia), CONACyT (Mexico), Ministry of Education and KOSEF (Korea), CONICET and UBACyT (Argentina), and the A.P. Sloan Foundation. 


\section{REFERENCES}

* Visitor from IHEP, Beijing, China.

$\dagger$ Visitor from Universidad San Francisco de Quito, Quito, Ecuador.

[1] CDF Collaboration, F. Abe et al., Phys. Rev. Lett. 74, 2626 (1995); DØ Collaboration, S. Abachi et al., Phys. Rev. Lett. 74, 2632 (1995).

[2] DØ Collaboration, S. Abachi et al., "Direct Measurement of the Top Quark Mass," Fermilab-PUB-97/059-E, submitted to Phys. Rev. Lett.

[3] DØ Collaboration, S. Abachi et al., Nucl. Instr. Meth. A 338, 185 (1994).

[4] D $\varnothing$ uses a cylindrical coordinate system about the beam axis, with polar angle $\theta$ and azimuthal angle $\phi$. The pseudorapidity $\eta \equiv \ln (\cot \theta / 2)$ is also used.

[5] DØ Collaboration, "Measurement of the Top Quark Pair Production Cross Section in $p \bar{p}$ Collisions," S. Abachi et al., Fermilab-PUB-97/109-E, submitted to Phys. Rev. Lett.

[6] E.W. Varnes, Ph.D. thesis, University of California, Berkeley (1997) (unpublished) (http://www-d0.fnal.gov/ publications_talks/thesis/thesis.html).

[7] K. Kondo, J. Phys. Soc. Jpn. 57, 4126 (1988) and 60, 836 (1991).

[8] R.H. Dalitz and G.R. Goldstein, Phys. Rev. D 451531 (1992) and Phys. Lett. B 287, 225 (1992).

[9] CTEQ Collaboration, H.L. Lai et al., Phys. Rev. D 51, 4763 (1995).

[10] G. Marchesini et al., Comp. Phys. Comm. 67, 465, v5.7 (1992).

[11] F. Paige and S. Protopopescu, BNL report No. BNL38034, v7.22 (1986) (unpublished).

$[12]$ T. Sjöstrand, Comp. Phys. Comm. 82, 74 (1994).

[13] L. Holmström, R. Sain, and H.E. Miettinen, Comp. Phys. Comm. 88, 195 (1995).

[14] DØ Collaboration, R. Kehoe, to be published in Proc. $6^{\text {th }}$ International Conf. on Calorimetry in High Energy Physics, Frascati (1996), Fermilab-Conf-96/284-E. 